\title{
Formation and optical characterization of single InAs quantum dots grown on GaAs nanoholes
}

\author{
P. Alonso-González, ${ }^{\text {a) }}$ B. Alén, D. Fuster, Y. González, and L. González \\ Instituto de Microelectrónica de Madrid (IMM-CNM, CSIC), Isaac Newton, 8 Tres Cantos, \\ Madrid 28760 Spain \\ J. Martínez-Pastor \\ Instituto de Ciencia de Materiales (ICMUV), Universidad de Valencia, P.O. Box 22085, \\ 46071 Valencia, Spain
}

(Received 28 August 2007; accepted 25 September 2007; published online 16 October 2007)

\begin{abstract}
We present a study of the structural and optical properties of InAs quantum dots formed in a low density template of nanoholes fabricated by droplet epitaxy on GaAs (001). The growth conditions used here promote the formation of isolated quantum dots only inside the templated nanoholes. Due to the good optical quality and low density of these nanostructures, their ensemble and individual emission properties could be investigated and related to the particular growth method employed and the quantum dot morphology. (C) 2007 American Institute of Physics. [DOI: 10.1063/1.2799736]
\end{abstract}

New nanoelectronics and quantum information perspectives require the ability of growing nanostructures with control in size and spatial location. In particular, for the application of quantum devices based in single quantum dots ${ }^{1,2}$ (QDs) as, for instance, single photon emitters, it is necessary to combine strategies that would permit precise location of a single nanostructure presenting at the same time high optical quality. $^{3-6}$

With this aim, a quite wide-spread strategy to overcome the randomly positioning of self-assembled nanostructures is based on the use of patterned substrates to create preferential nucleation sites that define the position and size of the nanostructures. In particular, highly ordered arrays of QDs have been already obtained using different lithographic approaches followed by an appropriate regrowth process. ${ }^{5,7}$ In this situation, the droplet epitaxy has revealed itself as a potential technique to achieve templates for localization of nanostructures with high optical quality in latticemismatched heteroepitaxial systems. ${ }^{9}$ This technique has been mainly developed in GaAs based systems and basically consists of the supply of a certain amount of Ga during growth to form metallic droplets on the surface, which are later transformed into crystalline GaAs under arsenic atmosphere. Under certain experimental conditions, the crystallization process leads to the formation of specific structures containing nanoholes that act as preferential sites for InAs QD nucleation upon further InAs deposition. ${ }^{10}$ The density of nanoholes corresponds to that of the droplets, being possible to obtain QD densities as low as $10^{7} \mathrm{~cm}^{-2}$. ${ }^{11}$ The size of these nanostructures is related to the amount of InAs filling the nanoholes, with independence of their density, as opposed to the QD formation by self-assembling processes. This fact is especially interesting for applications based on a single QD. Furthermore, in coincidence with other lithographic techniques, ${ }^{12}$ a different number of QD per nanohole can also be obtained. ${ }^{13}$ In this situation, one advantage of the droplet epitaxy technique is that it can provide nanotemplates for QD formation without the need of growing thick a) Author to whom correspondence should be addressed. Electronic mail:
palonso@imm.cnm.csic.es buffer layers to avoid the influence of residual contamination from the fabrication process on the optical properties of the nanostructures.

This work deals with the structural and optical characterization of InAs QDs formed in a template of 2.4 $\times 10^{8} \mathrm{~cm}^{-2}$ nanoholes built by droplet epitaxy on GaAs (001) substrates. We provide the growth procedure and analyze their typical ensemble photoluminescence (PL) spectra. Then, we study their emission properties at the single QD level to find that photon emission from negatively charged species $\left(X^{-n}\right)$ dominates over other recombination paths. This result is explained on the basis of the specific process that gives rise to the formation of our QDs on the droplet epitaxy templates.

Our particular process starts growing a $0.5 \mu \mathrm{m}$ thick undoped GaAs buffer layer at a substrate temperature $T_{s}=580{ }^{\circ} \mathrm{C}$ on GaAs (001) by molecular beam epitaxy. Then, the substrate temperature $T_{s}$ is decreased to $500{ }^{\circ} \mathrm{C}$ and the Ga shutter is opened during $10 \mathrm{~s}$ with the Ga cell, providing a flux equivalent to the growth of GaAs at $1 \mathrm{ML} / \mathrm{s}$. During this time, the arsenic cell is opened periodically during $0.2 \mathrm{~s}$ every $0.8 \mathrm{~s}$. The beam equivalent pressure (BEP) of $\mathrm{As}_{4}$ is fixed during the process to $5 \times 10^{-7}$ Torr. This process is a kind of arsenic-debt atomic layer molecular beam epitaxy growth ${ }^{14}$ and results in Ga droplets spread all over the surface with a density of $2.5 \times 10^{8} \mathrm{~cm}^{-2}$. The sample is then annealed during $6 \mathrm{~min}$ at the same substrate temperature and $\mathrm{As}_{4}$ pressure. Once the nanohole template is achieved, the substrate is heated to $510^{\circ} \mathrm{C}$ and $1.4 \mathrm{ML}$ of InAs are deposited following a growth sequence consisting of $0.1 \mathrm{ML}$ of InAs at a growth rate of $0.05 \mathrm{ML} / \mathrm{s}$ followed by a pause of $2 \mathrm{~s}$ under $\mathrm{As}_{2}$ flux $\left[\mathrm{BEP}\left(\mathrm{As}_{2}\right)=7.5\right.$ $\times 10^{-7}$ Torr]. After growth of a $100 \mathrm{~nm}$ thick GaAs cap layer, the process was repeated to obtain uncapped QD on the nanohole template for atomic force microscopy (AFM) measurements. For comparison, a reference sample with only nanoholes in the surface was grown skipping the InAs deposition step. The resulting structures appear with a density of $2.5 \times 10^{8} \mathrm{~cm}^{-2}$ and consist of two lobes that surround a nanohole. These structures are elongated along the [1-10] crystal direction with an average length of $250 \pm 15 \mathrm{~nm}$. The 

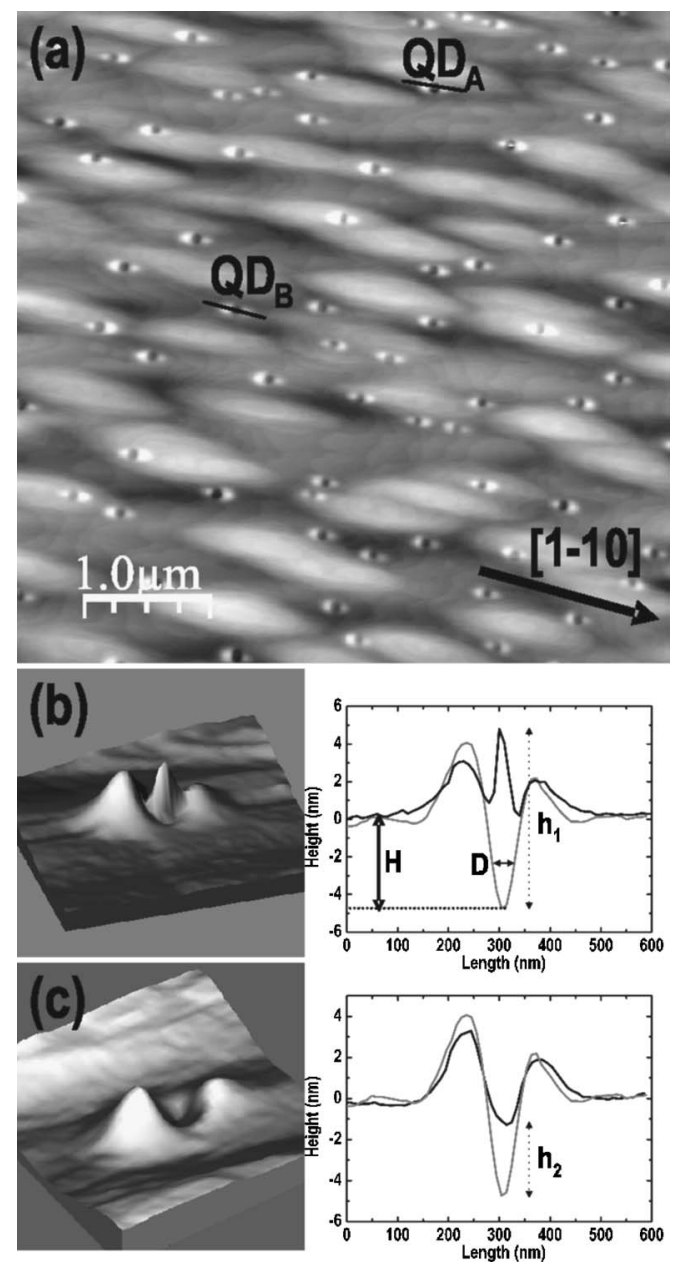

FIG. 1. AFM micrographs obtained in different areas of the sample are shown in (a) $\left(5 \times 5 \mu \mathrm{m}^{2}\right)$, and $[(\mathrm{b})$ and (c) $]\left(600 \times 600 \mathrm{~nm}^{2}\right)$. At the right, together with the typical QD profiles, a gray line has been drawn corresponding to the average profile of the GaAs nanoholes before InAs deposition.

nanoholes are $H=4.4 \pm 0.8 \mathrm{~nm}$ deep and have a mean diameter of $D=43 \pm 3 \mathrm{~nm}$ at their half minimum.

Figure 1 (a) shows a $5 \times 5 \mu \mathrm{m}^{2}$ AFM image of the QD sample surface. A profile along the nanostructure long axis reveals two different kinds of QDs labeled as A and B in the same figure. The differences are better observed on Figs. 1(b) and 1(c) where we depict typical images obtained on a smaller area and the corresponding [1-10] profiles of type-A and type-B nanostructures, respectively. In order to obtain the real dimensions of the InAs QDs, the profiles are compared with those obtained by averaging a large number of nanoholes from the reference sample [gray profiles on Figs. 1 (b) and 1(c)]. Type-A QDs are $h_{1}=9.0 \pm 1.8 \mathrm{~nm}$ high before capping, as measured from the bottom of the seeding nanohole. They are less frequent in the ensemble and appear with a density of $1 \times 10^{7} \mathrm{~cm}^{-2}$. On the other hand, type-B QDs [Fig. 1(c)] do not present the same topography as they do not protrude from its nanohole. Following the same procedure, we estimate their height to be $h_{2}=3.3 \pm 0.8 \mathrm{~nm}$. In this case, the density obtained for type-B QDs is $2.4 \times 10^{8} \mathrm{~cm}^{-2}$. At this point, we shall note that we did not find QDs in the regions in between the nanoholes, meaning that the growth conditions were optimal for formation of these nanostructures on patterned substrates. tures on patterned substrates.
Downloaded 31 Mar 2009 to 161.111.235.57. Redistribution subject to AlP license or copyright; see http://apl.aip.org/apl/copyright.jsp

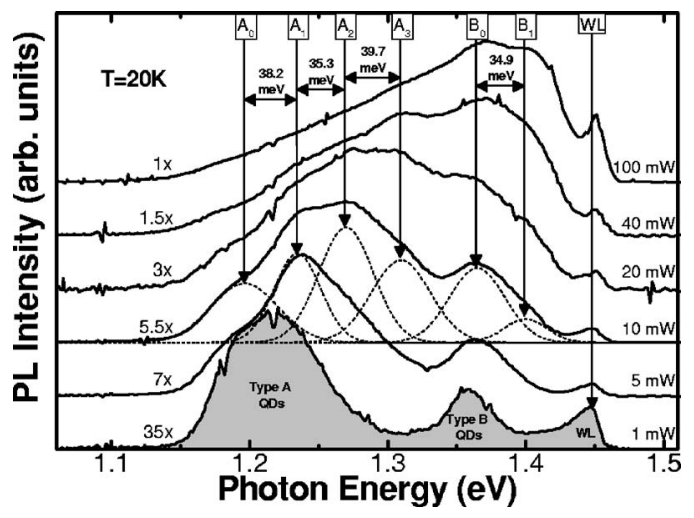

FIG. 2. The evolution with the excitation power of the ensemble PL spectrum reveals the existence of two different QD families and the emission from excited bands at the indicated energies. A Gaussian fit has been included for the spectrum obtained at $10 \mathrm{~mW}$.

Figure 2 shows the power dependent emission spectra obtained at $20 \mathrm{~K}$ using a standard PL setup with a spot diameter of $80 \mu \mathrm{m}$. At low excitation density, together with the wetting layer (WL) emission at $1.448 \mathrm{eV}$, we detected two broad bands at 1.196 and $1.357 \mathrm{eV}$ and labeled as $\mathrm{A}_{0}$ and $\mathrm{B}_{0}$, respectively. It seems clear that they correspond with the type-A and type-B QD families observed by AFM. At low temperatures, conventional InAs self-assembled QDs exhibit ground state energies around $1.1-1.18 \mathrm{eV}$ for capped QDs with heights in the range of 5-8 nm. ${ }^{15,16}$ On the other hand, introducing an annealing step during the growth or afterwards, their height decreases to $2-3 \mathrm{~nm}$ shifting up the ground state energy well above $1.3 \mathrm{eV} .{ }^{17}$ Therefore, we conclude that the height values found for type-A and type-B QDs are compatible with the fundamental transitions observed in their PL spectra.

We can also study the excited level structure for our QDs since, due to their low density, they enter in the saturation regime at moderate excitation powers. In Fig. 2, we identify three excited emission bands for type-A QDs peaked at 38.2, 73.5 , and $113.2 \mathrm{meV}$ above the ground state and labeled as $\mathrm{A}_{1}, \mathrm{~A}_{2}$, and $\mathrm{A}_{3}$, respectively. The approximately constant excited state splitting found indicates that the QD lateral confinement could be described by a smooth parabolic potential. ${ }^{18}$ The average value of $\Delta E \sim 38 \mathrm{meV}$ is smaller by $30 \%-50 \%$ than those reported in the literature for QDs emitting below $1.2 \mathrm{eV},{ }^{15,16}$ and it suggests, within this model, that type-A QDs exhibit a larger diameter than standing InAs/GaAs self-assembled QDs with similar ground state energy. Finally, for the type-B QD ensemble, we have found only one excited state $\left(\mathrm{B}_{1}\right)$ splitted by $34.9 \mathrm{meV}$ from its fundamental state in good correspondence with values reported in the literature for QDs emitting above $1.3 \mathrm{eV}$.

The emission properties at the single QD level have been investigated using a fiber based confocal microscope with a diffraction limited spot size running at $5 \mathrm{~K}$. The low density produced by the templated growth method allows the investigation of only one QD at a time, as shown in Fig. 3. We have studied several QDs of the type-B family throughout the sample surface (detector cutoff at $\sim 1000 \mathrm{~nm}$ ) finding similar groups of spectral lines for all of them. Increasing the laser excitation at the WL band edge, the biexciton recombination peak $\left(X X^{0}\right)$ can be easily identified from its superlinear dependence on excitation power. At the same time, at low 

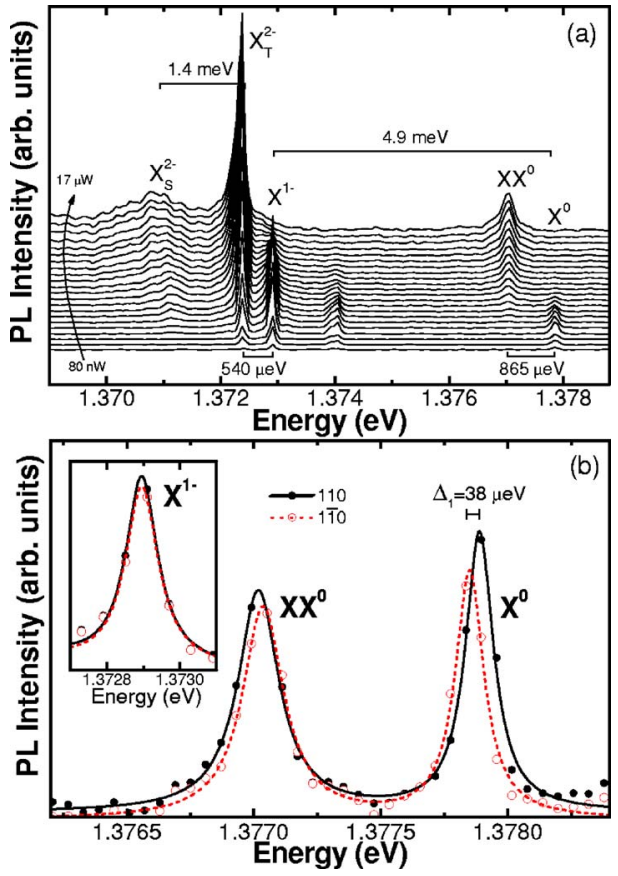

FIG. 3. (Color online) (a) Microphotoluminescence spectra of a single type-B QD measured for increasing excitation intensities. The emission peaks corresponding to different excitonic species have been identified by their power and polarization behavior as discussed in the text ( $S$ and $T$ mean singlet and triplet, respectively). (b) Polarized spectra recorded for the same single QD along perpendicular crystal directions showed the splitting of the neutral states.

spectrum as the excitation intensity is raised. The relative splittings found among the different $\mu$-PL lines are characteristic of negatively charged exciton recombination in QDs of this size. ${ }^{18}$ Yet, this assignment can be further confirmed by measuring the polarization resolved spectrum for each line, as shown in Fig. 3(b). As expected, the $X^{0}$ and $X X^{0}$ peaks experience opposite shifts upon $90^{\circ}$ turn of the linear polarization analyzer aligned along the [1-10] crystal direction. The maximum splitting of $\Delta_{1} \sim 38 \mu \mathrm{eV}$ is due to the anisotropic exchange interaction between electrons and holes in slightly elongated QDs. Since the interaction is suppressed due to the singlet nature of the singly charged exciton $\left(X^{1-}\right)$, this fact can be used to identify its emission peak, as shown in the inset of the same figure. The rather small value found for $\Delta_{1}$ indicates an approximately round in-plane confinement potential which makes these nanostructures interesting for applications based on the generation of polarization entangled photon pairs. ${ }^{1}$

Finally, to explain the dominant role of negatively charged species recombination in our sample, we shall note that the proposed templated growth method is based on the deposition of metallic Ga droplets. If the crystallization process is incomplete, a number of arsenic vacancies will remain in the surroundings of the QD. These defects are known to produce localized states near the conduction band which can easily transfer their electrons to the QDs. ${ }^{19} \mathrm{Sev}-$ eral recent works explain how, under selective optical pumping ${ }^{20,21}$ or increasing the excitation power, ${ }^{22,23}$ this situation will lead to preferential emission from negatively charged exciton species as we observe here. This fact must be considered in future developments based in this kind of templated nanostructures.
In summary, the growth of InAs QDs on GaAs (001) using a template formed by droplet homoepitaxy has been demonstrated. The method produces QDs with two different heights that have been investigated by structural and optical means. A relatively large lateral size has been proposed to explain the rather small splitting found between electronic shells for the larger QDs. For the smaller QDs, the investigation of their $\mu$-PL spectra has revealed an approximately round lateral morphology and the presence of arsenic vacancies in the QD surroundings due to the incomplete droplet crystallization. The good optical quality and templated nature of these nanostructures make them potential candidates for single quantum devices and applications.

The authors gratefully acknowledge the financial support by the Spanish MEC and CAM through project Nos. TEC2005-05781-C03-01/-03 and NAN2004-09109-C04-01/-03, by the Consolider-Ingenio 2010 CSD2006-0019 and S-505/ ESP/000200, and by the European Commission through SANDIE Network of Excellence (No. NMP4-CT-2004500101). P.A.G. thanks the I3P program.

${ }^{1}$ R. M. Stevenson, R. J. Young, P. Atkinson, K. Cooper, D. A. Ritchie, and A. J. Shields, Nature (London) 439, 179 (2006).

${ }^{2}$ A. Badolato, K. Hennessy, M. Atatüre, J. Dreiser, E. Hu, P. M. Petroff, and A. Imamouglu, Science 308, 1158 (2005).

${ }^{3}$ S. Kiravittaya, M. Benyoucef, R. Zapf-Gottwick, A. Rastelli, and O. G. Schmidt, Appl. Phys. Lett. 89, 233102 (2006).

${ }^{4}$ E. Pelucchi, S. Watanabe, K. Leifer, Q. Zhu, B. Dwir, P. De Los Rios, and E. Kapon, Nano Lett. 7, 1282 (2007)

${ }^{5}$ P. Atkinson, M. B. Ward, S. P. Bremner, D. Anderson, T. Farrow, G. A. C. Jones, A. J. Shields, and D. A. Ritchie, Jpn. J. Appl. Phys., Part 1 45, 2519 (2006).

${ }^{6}$ H. Z. Song, T. Usuki, S. Hirose, K. Takemoto, Y. Nakata, N. Yokoyama, and Y. Sakuma, Appl. Phys. Lett. 86, 113118 (2005).

${ }^{7}$ J. Martín-Sánchez, Y. González, L. González, M. Tello, R. García, D. Granados, J. M. García, and F. Briones, J. Cryst. Growth 284, 313 (2005).

${ }^{8}$ P. Alonso-González, L. González, Y. González, D. Fuster, I. Fernández-Martínez, J. Martín-Sánchez, and L. Abelmann, Nanotechnology 18, 355302 (2007).

${ }^{9}$ T. Mano, K. Watanabe, S. Tsukamoto, H. Fujioka, M. Oshima, and N. Koguchi, J. Cryst. Growth 209, 504 (2000).

${ }^{10}$ Zh. M. Wang, B. L. Liang, K. A. Sablon, and G. J. Salamo, Appl. Phys. Lett. 90, 113120 (2007).

${ }^{11}$ C. Zhao, Y. H. Chen, B. Xu, P. Jin, and Z. G. Wang, Appl. Phys. Lett. 91, 033112 (2007).

${ }^{12}$ R. Songmuang, S. Kiravittaya, and O. G. Schmidt, Appl. Phys. Lett. 82, 2892 (2003).

${ }^{13}$ J. H. Lee, Zh. M. Wang, N. W. Strom, Yu. I. Mazur, and G. J. Salamo, Appl. Phys. Lett. 89, 202101 (2006).

${ }^{14}$ F. Briones, L. González, and A. Ruiz, Appl. Phys. A: Solids Surf. 49, 729 (1989).

${ }^{15}$ S. Raymond, S. Fafard, P. J. Poole, A. Wojs, P. Hawrylak, and S. Charbonneau, Phys. Rev. B 54, 11548 (1996).

${ }^{16}$ M. Grundmann, N. N. Ledentsov, O. Stier, J. Böhrer, D. Bimberg, V. M. Ustinov, P. S. Kop'ev, and Zh. I. Alferov, Phys. Rev. B 53, 10509 (1996).

${ }^{17}$ A. Greilich, M. Schwab, T. Berstermann, T. Auer, R. Oulten, D. Yakovlev, M. Bayer, V. Stavarache, D. Reuter, and A. Wieck, Phys. Rev. B 73, 045323 (2006).

${ }^{18}$ B. Alén, J. Martínez-Pastor, D. Granados, and J. M. García, Phys. Rev. B 72, 155331 (2005).

${ }^{19}$ H. Q. Xu and U. Lindefelt, Phys. Rev. B 41, 5979 (1990).

${ }^{20}$ E. S. Moskalenko, K. F. Karlsson, P. O. Holtz, B. Monemar, W. V. Schoenfeld, J. M. Garcia, and P. M. Petroff, Phys. Rev. B 64, 085302 (2001).

${ }^{21}$ G. Muñoz-Matutano, B. Alén, J. Martínez-Pastor, L. Seravalli, P. Frigeri, and S. Franchi, e-print arXiv:cond-mat/0704.2319v1.

${ }^{22}$ A. Hartmann, Y. Ducommun, E. Kapon, U. Hohenester, and E. Molinari, Phys. Rev. Lett. 84, 5648 (2000).

${ }^{23}$ D. V. Regelman, E. Dekel, D. Gershoni, E. Ehrenfreund, A. J. Williamson, J. Shumway, A. Zunger, W. V. Schoenfeld, and P. M. Petroff, Phys. Rev. B 64, 165301 (2001). 